\title{
Dynamic regulation of DNA nanostructures by noncanonical nucleic acids
}

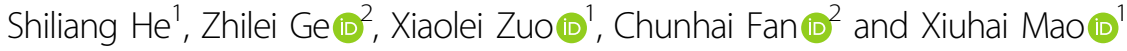

\begin{abstract}
DNA nanostructures are among the most fascinating self-assembled nanostructures in diverse areas of science and technology, because of their nanoscale precision in biomolecule and nanoparticle organization. The implementation of dynamic and spatial regulation in structural morphology and hierarchical assembly upon specific external stimuli will greatly expand their applications in biocomputation, clinical diagnosis, and cancer therapy. Recently, noncanonical nucleic acids, particularly DNA triplexes, i-motifs, and G-quadruplexes, have become powerful tools for biosensing and mechanical switching. Developments in incorporating stimuli-responsive noncanonical nucleic acids into DNA nanostructures provide a promising approach to regulating the spatial organization and hierarchical assembly of DNA nanostructures. In this review, we briefly introduce recent progress in constructing DNA nanostructures with dynamic regulation of the structural transformation and programmable assembly pathways at the nanometer scale by noncanonical nucleic acids and discuss their potential applications and challenges.
\end{abstract}

\section{Introduction}

Since Nardaran Seeman first constructed artificial DNA nanostructures in the $1980 \mathrm{~s}^{1}$, well-defined nanoscale DNA architectures have been designed and fabricated, including cubes $^{2}$, tetrahedrons ${ }^{3}$, polyhedra ${ }^{4}$, DNA origami $^{5,6}$, and spherical nucleic acids (SNAs) ${ }^{7}$ (Fig. 1a). Owing to their nanoscale precision in organizing and manipulating molecules, these DNA nanostructures have attracted intense and widespread interest in cutting-edge research areas of physiological studies, clinical diagnosis, cancer therapy, and biocomputing ${ }^{8-10}$. Currently, developments in harnessing the structural and material properties of DNA nanostructures, including their size at different scales, topologic shape, and spatial arrangement, have been achieved. The next major challenge in DNA

\footnotetext{
Correspondence: Chunhai Fan (fanchunhai@sjtu.edu.cn) or

Xiuhai Mao (maoxiuhai@sjtu.edu.cn)

'Institute of Molecular Medicine, Shanghai Key Laboratory for Nucleic Acid Chemistry and Nanomedicine, Renji Hospital, School of Medicine and School of Chemistry and Chemical Engineering, Shanghai Jiao Tong University, Shanghai, China

${ }^{2}$ School of Chemistry and Chemical Engineering, Frontiers Science Center for Transformative Molecules, Institute of Translational Medicine, Shanghai Jiao Tong University, Shanghai, China
}

nanostructure functionalization is the dynamic regulation of their structural transformation and hierarchical assembly. In particular, reconfigurable and dynamic DNA nanostructures, which can be subjected to on-demand regulation of the morphology, motion and, material properties in response to various external stimuli, play a critical role in a spectrum of fields, including nanorobotics, nanophotonics, nanosensors, nanoelectronics, and nanomedicine ${ }^{11,12}$. To this end, many efforts have been made in recent years to develop bottom-up methods for functionalizing DNA nanostructures to carry out their structural reconfiguration or manipulate assembly behavior with programmed tunability, revertability and specificity in molecular recognition ${ }^{13,14}$.

In addition to the right-handed B-form double helix, a variety of nucleic acid conformations that disobey the Watson-Crick canon, particularly the DNA triplex ${ }^{15}$, G-quadruplex ${ }^{16}$, and i-motif (Fig. 1b) ${ }^{17}$, are highly attractive functional modules in constructing dynamic and sophisticated DNA nanostructures. Specifically, the DNA triplex is self-assembled by a DNA duplex hybridizing with a third ssDNA through either Hoogsteen or reverse Hoogsteen hydrogen bonding, with preferred base triplets including 


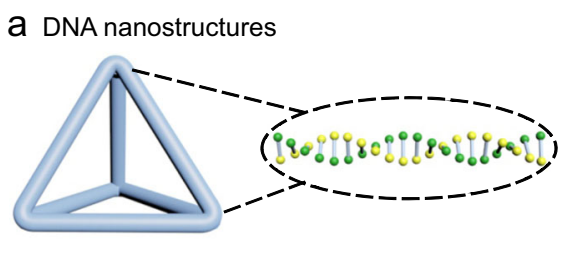

Tetrahedral DNA

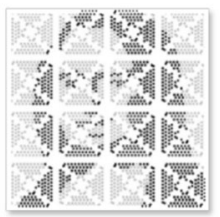

DNA origami array

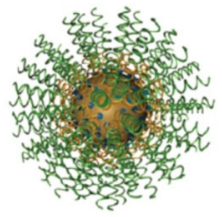

SNA

b Noncanonical nucleic acids

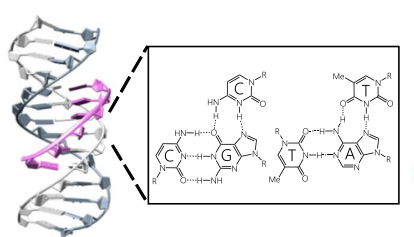

Triplex

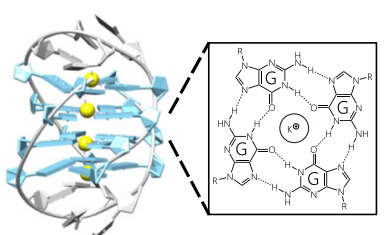

G-quadruplex

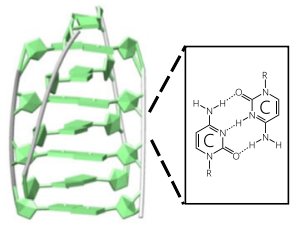

i-motif

C Regulation via conformational switching of noncanonical nucleic acids

Structural regulation of a nanostructure: Hierarchical assembly regulation:
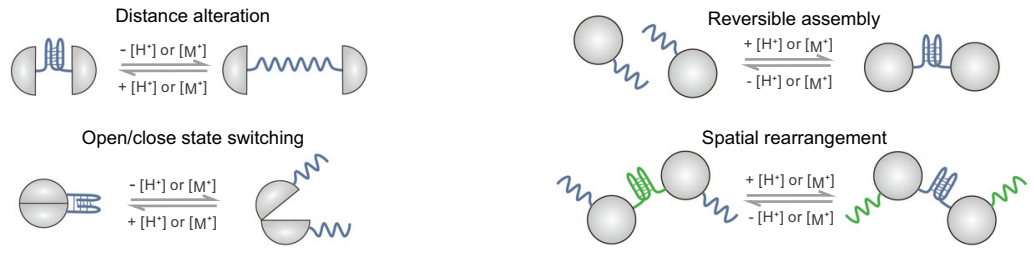

Fig. 1 Dynamic regulation of artificial DNA nanostructures with noncanonical nucleic acids. a Representative structures of artificial DNA nanostructures, including a tetrahedral DNA, a DNA origami array with a pattern of Mona Lisa (reproduced with permission ${ }^{6}$, Copyright 2018, Elsevier) and spherical nucleic acids (reproduced with permission7, Copyright 2012, American Chemical Society). b Representative conformations of noncanonical nucleic acids. (left) X-ray structure of a parallel triplex (PDB ID: 1BWG) composed of CH + G-C and T-A-T triads. The two duplex-forming strands were colored, respectively, light and dark gray, while the triplex-forming oligonucleotide (TFO) was highlighted in purple. (middle) X-ray structure of a bimolecular G-quadruplex (PDB ID: 1JB7) assembled from two d(GGGGTTTTGGGG) oligonucleotides. The guanosines were colored blue and four potassium cations yellow. (right) X-ray structure of a tetramolecular i-motif viewed from the major groove side (PDB ID: 190D), which was assembled from four $\mathrm{d}(\mathrm{CCCC})$ oligonucleotide. Hemiprotonated $\mathrm{CH}+-\mathrm{C}$ base pair was formed between two parallel strands. The three crystal figures were drawn with UCSF Chimera. c Regulation mechanisms of noncanonical nucleic acids on DNA nanostructures. A complete gray ball represents a DNA nanostructure of all sorts, and blue/green lines represent noncanonical DNA.

$\mathrm{T} \cdot \mathrm{A}-\mathrm{T}, \mathrm{A} \cdot \mathrm{A}-\mathrm{T}, \mathrm{G} \cdot \mathrm{G}-\mathrm{C}$, and $\mathrm{CH}^{+} \cdot \mathrm{G}-\mathrm{C}$. G-quadruplexes are formed by alkali-metal-assisted stacking of guanine (G) tetrads held by Hoogsteen base pairs, while i-motifs adopt cytosine $(\mathrm{C})$-cytosinium $\left(\mathrm{CH}^{+}\right)$base pairs resulting from the protonation of $\mathrm{N}-3$ on cytosine. The difference in their structural formation requirements from their duplex counterparts brings about novel capacity in manipulating DNA nanostructures in many ways that would not have been possible by using solely DNA duplexes.

Taking advantage of DNA nanotechnology, researchers have successfully designed and assembled a series of noncanonical nucleic acid-incorporated DNA nanostructures with dynamic regulation in structural transformation and hierarchical assembly (Fig. 1c and Table 1). For instance, by embedding noncanonical nucleic acids into the structures of individual DNA constructs, the distance between certain components can be adjusted, thereby altering the morphology or configuration of the
DNA constructs. Moreover, reversible assembly/disassembly of the DNA nanostructures, as well as their spatial rearrangement, can be achieved. In this review, we focus on new progress in utilizing noncanonical nucleic acids to dynamically regulate the structural reconfiguration and hierarchical assembly of artificial DNA nanostructures, which hold wider implications for expanding and regulating the functionalities of materials beyond nucleic acids. Furthermore, we discuss the respective roles and interplay of artificial DNA nanostructures and naturally occurring noncanonical nucleic acids in various applications, including nanosensors, nanomedicine and, nanophononics.

\section{Reconfigurable elements for regulating DNA nanostructures}

Noncanonical nucleic acid conformations have been characterized in vitro with various methods, such as fiber 
Table 1 Overview of DNA nanostructures regulated with noncanonical nucleic acids.

\begin{tabular}{|c|c|c|c|c|}
\hline Noncanonical nucleic acid & DNA nanostructure & Functionality & Application & Ref. \\
\hline \multirow[t]{4}{*}{ Triplex } & \multirow[t]{2}{*}{$2 \mathrm{D}$ origami } & Structural isomerization depending on $\mathrm{pH}$ & Programmed assembly & 32 \\
\hline & & Hierarchical assembly depending on $\mathrm{pH}$ & Biomimetic assembly & 68 \\
\hline & 3D origami & Opening/closing of origami capsule depending on $\mathrm{pH}$ & Drug delivery & 64 \\
\hline & SNA & Releasing drug-loaded SNA in acidic tumor microenvironment & Drug delivery & 70 \\
\hline \multirow[t]{5}{*}{ i-Motif } & \multirow[t]{3}{*}{ DNA tetrahedron } & Cavity volume regulation depending on $\mathrm{pH}$ & Nano-pump & 52 \\
\hline & & Opening/closing of DNA cage depending on pH & Catalysis & 63 \\
\hline & & Dimerization depending on $\mathrm{pH}$ and ATP & Logic gate/ATP imaging & 31 \\
\hline & DNA triangular prism & FRET pair distance change in acidic organelles & $\mathrm{pH}$ sensor & 58 \\
\hline & 2D origami & Distance alteration between substructures depending on $\mathrm{pH}$ & Nano-actuator & 51 \\
\hline \multirow[t]{5}{*}{ G-quadruplex } & DNA triangle & Dimerization depending on $\mathrm{K}^{+}$ & Catalysis & 30 \\
\hline & \multirow[t]{2}{*}{ 2D origami } & Interorigami distance alteration by UV irradiation & Optical reporter & 38 \\
\hline & & Structural rearrangement depending on $\mathrm{K}^{+}$ & Catalysis & 33 \\
\hline & \multirow[t]{2}{*}{ 3D origami } & Curvature change depending on $\mathrm{K}^{+}$ & Nano-motor & 40 \\
\hline & & Fluorescence change by $\mathrm{K}^{+}$-responsive structural change & $\mathrm{K}^{+}$sensor & 59 \\
\hline
\end{tabular}

diffraction, NMR spectra, and X-ray crystallography ${ }^{18}$, and have been visualized recently in human cells ${ }^{19-21}$. In addition to executing regulatory functions in various cellular processes, including DNA replication, transcription, and recombination ${ }^{19,22}$, they have been widely utilized in the DNA nanotechnology field as reconfigurable elements, owing to their peculiar conformations, properties, and functionalities.

First, noncanonical nucleic acids exhibit a high degree of conformational polymorphism, enabling the fabrication of DNA nanostructures with outstanding structural complexity and diversity. For instance, the strand molecularity of noncanonical nucleic acids varies from 1 to 4 . Both triplex and tetraplex nucleic acids can be formed by the assembly of one, two, three, or four oligonucleotide strands ${ }^{23-29}$, existing as either intra- or intermolecular conformations. The commonly applied conformational switching strategies are summarized in Fig. 2: (a) Intramolecular conformational switching of monomeric triplex/tetraplex DNA. (b) Intermolecular conformational switching of a dimeric triplex/ tetraplex. In this strategy, short duplex or hairpin DNA is incorporated into the intertriplex/intertetraplex conformation to enhance its thermodynamic stability and to prevent the formation of undesired structures ${ }^{30,31}$. (c) Switching between intramolecular and duplexed conformations by introducing a strand with partial complementarity to the triplex/tetraplex-forming strand. This strategy possesses inherent hybridization, directionality and benefits, and the dictation of programmable assembly ${ }^{32,33}$.

Moreover, the thermodynamic stabilities of noncanonical nucleic acids are determined by their sequence compositions and lengths ${ }^{30,31,34}$. This feature provides immense room for the rational design of noncanonical nucleic acid-based functional modules with on-demand performance in hybridization. The formation of the G-quadraplex or i-motif requires four stretches of at least two consecutive $\mathrm{Gs}$ or $\mathrm{Cs}^{35-37}$. Stretches of six or more G- or C-residues are long enough to fold back on themselves, making it possible for them to fold into either of the two conformations (i.e., intra- and intermolecular) in a tetraplex structure. For instance, oligonucleotide $\mathrm{C}_{6} \mathrm{TC}_{6}$ folds into an intermolecular tetraplex structure, while $\mathrm{C}_{6} \mathrm{~T}_{3} \mathrm{C}_{6}$, $\mathrm{C}_{7} \mathrm{TC}_{7}$, and $\mathrm{C}_{7} \mathrm{~T}_{3} \mathrm{C}_{7}$-fold into intramolecular conformations $^{27}$. Thus, sequences consisting of 3-5 consecutive Gs or Cs are commonly used in DNA nanotechnology ${ }^{37-40}$. Triplexes can form only with at least five TAT base triplets in their stems at room temperature, with longer stems giving generally higher stability ${ }^{41}$.

Noncanonical nucleic acids can also accommodate a range of folding topologies. G-quadruplexes manifest at best topological complexity: intramolecular DNA G-quadruplexes can theoretically adopt 26 folding topologies, classified based on a number of interdependent structural parameters, including the glycosidic bond configuration, groove width, and loop position ${ }^{42}$. In terms of the relative orientations for adjacent strands, either two, three or all four strands in a G-quadruplex can be oriented in the same direction, while the rest, in the opposite direction. For dimeric i-motif DNA, the length of C-tracts determines the topology to be either head-to-head or head-to-tail (classified based on the relative position of the two loops $)^{37}$. Once the $\mathrm{C}$-tracts contain an unequal 


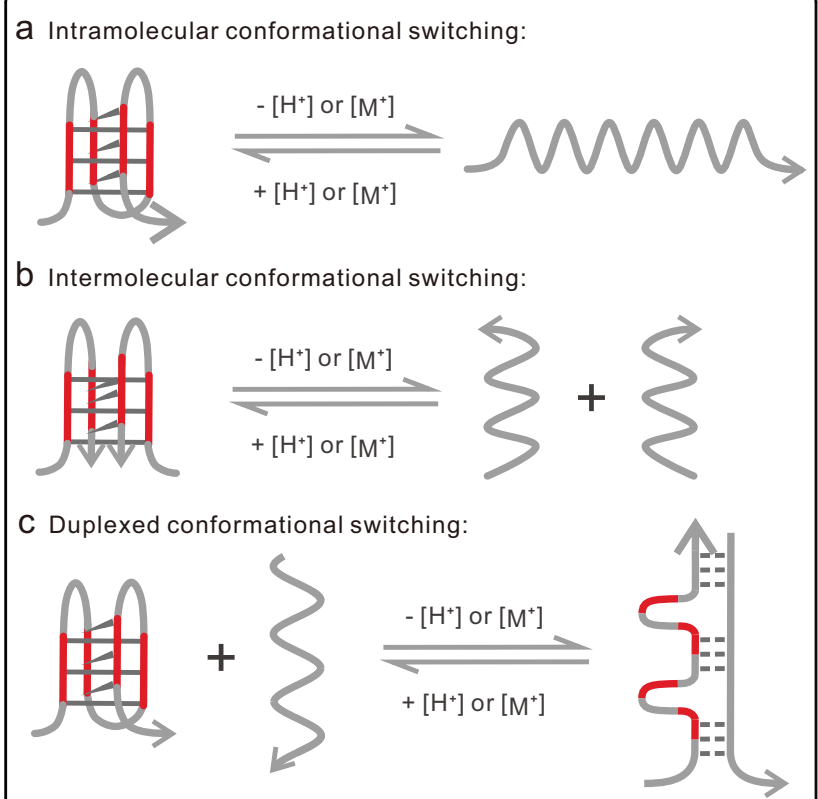

Fig. 2 Three strategies of inverting noncanonical DNA conformations upon proton or metal ions stimulation. a Intramolecular conformational switch of a monomeric triplex/tetraplex DNA. b Intermolecular conformational switch of a dimeric triplex/ tetraplex DNA. c Switch between intramolecular and duplexed conformations by introducing a strand with partial complementarity to the triplex/tetraplex-forming strand. The arrows of the strands indicate the direction of oligonucleotides from $5^{\prime}$ to $3^{\prime}$. Strand fragments of forming noncanonical nucleic acids are colored red.

number of C-residues, only the head-to-head arrangement is thermodynamically favored ${ }^{37}$. In triplexes, the third strand can bind to the duplex motif in either parallel or antiparallel orientation with respect to the central strand, with the parallel topology exhibiting higher thermal stability and less tendency to form mismatches ${ }^{43}$.

In addition, given that the formation of noncanonical nucleic acids depends on external stimuli, including $\mathrm{pH}^{34,44}$, temperature ${ }^{25,45}$, ionic strength ${ }^{26,46}$, and noble metals ${ }^{47}$, this stimuli responsiveness greatly enriches the regulatory mechanism of the ever-developing DNA nanotechnology field. For example, the applicable $\mathrm{pH}$ ranges of the triplex and i-motif are $\mathrm{pH} 4.5-10$ and pH 5-7.4, respectively ${ }^{32,34,44,48}$. They are able to fold at salt concentrations ranging from physiological to high, nonphysiological levels (e.g., $10 \mathrm{mM} \mathrm{MgCl}_{2}$ or $315 \mathrm{mM}$ $\mathrm{KCl})^{45,49}$. G-quadruplexes are stabilized optimally by potassium ions $\left(\mathrm{K}^{+}\right)$, and the applicable $\mathrm{K}^{+}$concentration range is $\sim 0.1-1000 \mathrm{mM}^{46}$. Notably, because these environmentally friendly stimuli can be readily implemented in the laboratory to induce DNA conformational switching $^{50}$, the three DNA conformations (triplex, i-motif, and G-quadraplex) have been more frequently adopted for functionalizing or regulating DNA nanostructures than other non-B DNA, including left-handed Z-DNA, poly (dA) duplex (A-motif) and DNA bearing $\mathrm{T}-\mathrm{Hg}-\mathrm{T}$ base pairs. Their favorable thermodynamic stability under physiological conditions further ensures their application in cellular environments.

\section{Structural regulation of individual DNA nanostructures}

With the help of precise addressability and programmability, 2D or 3D nanostructures with different sizes and geometries have been deliberately designed ${ }^{8-10}$. When stimuli-responsive noncanonical nucleic acids are incorporated into the molecular structure of these DNA nanostructures, various external stimuli can be used to regulate their structural properties. Specifically, they are subjected to transition among two or more distinct structural states in which the spatial arrangement of their structural components is nonidentical. This structural regulation leads to nano- or macroscale changes in their morphologies, which can be unambiguously visualized by imaging techniques ${ }^{13}$. The mechanical force generated in this process also realizes internal movement in individual nanostructures, which is useful for constructing actuators implementable within the DNA nanotechnology toolkit ${ }^{12}$. Here, we highlight two research areas in the structural regulation of individual DNA nanostructures, including regulation of the distance and direction between specific components at the nanoscale and the spatial organization of non-DNA molecules tethered to the DNA nanostructure, which may lead to extraordinary functional nanodevices.

\section{Dynamic structural regulation of DNA nanostructures}

The fast-growing field of DNA nanodevices, which utilize DNA nanostructures to localize, guide, and regulate biomaterials at the nanometer scale, is an emerging research area ${ }^{8,11}$. The structural reconfiguration of DNA nanostructures could directly and substantially influence their material properties ${ }^{12,13}$. Noncanonical nucleic acids are powerful tools in transforming their conformations to generate mechanical force, thereby regulating the spatial relative distance and position of components of DNA nanostructures ${ }^{50}$.

When noncanonical DNA of intramolecular conformation is embedded between two bound subunits in a DNA nanostructure, they can function as distance or angle adjusters by transitioning between the folded and unfolded states to execute motions. For example, the i-motif is compact and rigid in its folded state, with a width of $\sim 0.8 \mathrm{~nm}$, while in the open state, it is a single strand of DNA with an end-to-end distance that is a few nanometers longer. This property was utilized to measure motions between two origami units both in solution and on the surface ${ }^{51}$. Specifically, after accommodating multiple (CCCTAA) ${ }_{3} \mathrm{CCC}$ strands in parallel at the seam of 


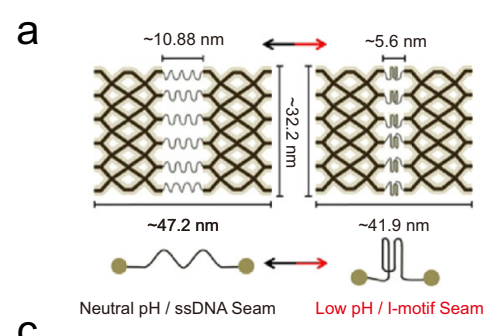

C
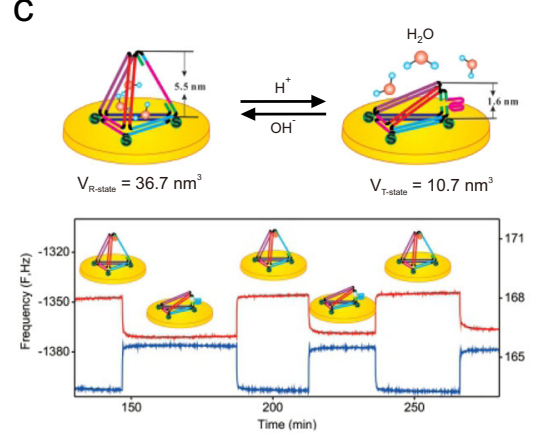

b



d

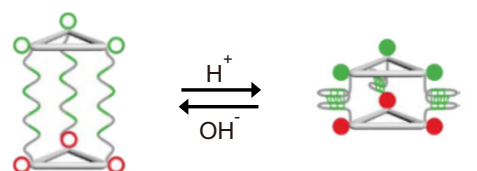

FRET OFF

Extracellular $\mathrm{pH} \sim 7.4$ / Cytoplasm pH 7.0

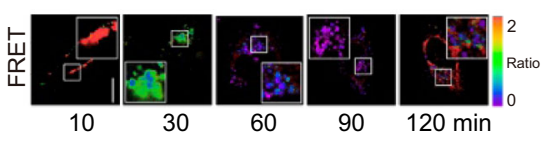

Fig. 3 Regulation of DNA nanostructures via relative distance alteration between their components induced by noncanonical DNA conformation switching. a A monomeric i-motif-bridged DNA origami pH-driven actuator. Reproduced with permission ${ }^{51}$. Copyright 2017, American Chemical Society. b Monomeric G-quadruplexes-induced reversible large deformation of a DNA origami nanoarm. AFM images of the origami before and after $\mathrm{K}+$ treatment were inserted. Scale bars: $200 \mathrm{~nm}$. Reproduced with permission ${ }^{40}$. Copyright 2020, Wiley-VCH. c A protondriven nanopump based on monomeric i-motif-integrated DNA tetrahedrons. Curves acquired with quartz crystal microbalance were shown below the scheme. Reproduced with permission ${ }^{52}$. Copyright 2016, Wiley-VCH. d Intracellular pH sensor based on monomeric i-motif-functionalized DNA triangular prism. The green and red circles denote Cy3 and Cy5, respectively. Spatiotemporal pH mapping of HeLa cells was shown below at a series of time points. Reproduced with permission ${ }^{58}$. Copyright 2019, American Chemical Society.

two bound origami, $\sim 3 \mathrm{~nm}$ variation in distance was observed by subjecting the mica surface first to a $\mathrm{pH} 7.2$ buffer and then a pH 5.5 buffer, and vice versa (Fig. 3a).

Similarly, the curvature of the DNA nanostructure could also be reversibly altered by incorporating a monomeric noncanonical nucleic acid in the middle of duplex scaffolds. For example, a DNA origami nanoarm was constructed with 19 repeats of a tension-adjustable module, each comprising, a stem, piers, and bridge strands (Fig. 3b). The bridge strands connecting the piers acted as entropic springs to apply tension to bend the entire nanoarm. Because a G-quadruplex-forming sequence was incorporated into the bridge strand, it contracted in the presence of $\mathrm{K}^{+}$, thereby leading to the bending of the module and subsequently the deformation of the entire shape from the original linear shape into an arched shape in a cumulative fashion ${ }^{40}$.

Relative movements between components of a DNA nanostructure lead to alterations in volume when it bears a cavity in its structure. This feature was readily illustrated by the DNA tetrahedron, as it is probably the simplest and most accessible 3D DNA cage. By inserting a singlestranded i-motif-forming sequence into one of the edges of a DNA tetrahedron immobilized on a gold surface, our group demonstrated proton-driven uptake and extrusion of water and ions in/from the tetrahedron inner cavity, resembling the expansion and compression of a water pump (Fig. 3c) ${ }^{52}$. The weight change of entrapped water caused by the conformational switching of the surfaceconfined DNA tetrahedron was measured using a quartz crystal microbalance (QCM). When PBS buffers with $\mathrm{pH}$ values of 8.0 and 5.0 were alternatively pumped into the QCM chamber, reversible conformational switching could be monitored by the change in both the frequency (blue) and energy dissipation (red) curves acquired with the quartz crystal microbalance.

Sensing is another important application field of reconfigurable DNA nanostructures modified with noncanonical DNA. DNA nanostructures of different shapes and compositions are able to serve as scaffolds or carriers for noncanonical DNA-based sensors in specific working scenes both in vivo and in vitro. The intrinsic dependence on protonation in the folding of the DNA triplex and $\mathrm{i}$-motif endows them with the power to sense $\mathrm{pH}$ variations. Cells and organelles elaborately maintain appropriate proton gradients to function properly, and abnormal $\mathrm{pH}$ alterations have a substantial influence on multiple important cellular processes, such as apoptosis and cell proliferation, which lead to the development and progression of cancer $^{53}$. Gaining knowledge of $\mathrm{pH}$ alteration and the exact value in biological media will therefore contribute to the understanding and 
interference of these processes ${ }^{54,55}$. Moreover, biosensors with G-quadruplexes as the target capture elements are able to detect potassium ions, which play a significant role in controlling cellular osmotic pressure and whose concentration abnormalities in blood are associated with heart disorders and lethal complications ${ }^{56}$. In addition, G-quadruplex-forming oligonucleotides comprise a large group of aptamers that recognize ions $\left(\mathrm{Cu}^{2+}, \mathrm{Pb}^{2+}\right.$, etc.), small molecules (ATP, ochratoxin A, etc.), and proteins (thrombin, nucleolin, immunoglobulin $\mathrm{M}$, etc.) ${ }^{57}$.

A straightforward approach to functionalizing DNA nanostructures with the sensing ability is to integrate a monomeric triplex/tetraplex into their structures, with fluorophore-quencher or FRET pairs modified at its two terminals, so that the generation of a fluorescent signal and its relative intensity will exhibit dependence on the conformational transition of the integrated triplex/tetraplex. In a recent report, a DNA triangular prism was selfassembled to include i-motif-forming sequences in its three edges, and three identical FRET pairs were modified on both ends (Fig. 3d) ${ }^{58}$. The FRET signal was activated when the DNA prism entered acidic organelles, owing to intramolecular i-motif folding, which brought the FRET pairs into close proximity, while in neutral $\mathrm{pH}$ conditions, the DNA prism extended and diminished the FRET effect. High contrast and spatiotemporal $\mathrm{pH}$ mapping were thus achieved during endocytosis. In addition, instead of placing recognition elements directly between FRET pairs, another approach places the G-quadraplex-forming strand at a distance from the FRET pair on tunable DNA origami to regulate $\mathrm{K}^{+}$-induced signal generation ${ }^{59}$.

Artificial DNA nanostructures, including tetrahedral DNA, SNA, and DNA origami, have been employed recently as scaffolds to fabricate G-quadruplex-containing-aptamer-based sensors and drug delivery vehicles ${ }^{60-62}$. For example, in the design of an electrochemical sensor for thrombin detection, the binding of target thrombin to its G-rich aptamer, which relies on a G-quadruplex structural transformation, opened hairpins conjugated on a polymeric ferrocene nanoparticle, releasing a singlestranded region for further hybridization with hairpins on the vertex of DNA tetrahedrons modified on the electrode surface $^{61}$. Strand displacement released the target thrombin for the next cycle of capturing nanoparticles. Each copy of the target thrombin was in principle capable of inducing uncountable cycles to enrich the signal enhancer molecule ferrocene on the electrode, resulting in superior detection sensitivity.

In another case of inducing selective tumor cell death by the occlusion of tumor blood vessels, precise delivery of the blood coagulation protease thrombin to tumor sites was achieved with DNA origami, capable of reconfiguration between the planar sheet state and closed nanotube $\operatorname{state}^{62}$. The thrombin molecules were anchored to the inner surface of the origami sheet. The seam of the origami nanotube was functionalized with G-quadruplex-containing aptamers that bind nucleolin, a protein specifically expressed on tumor-associated endothelial cells. The origami nanotube was noncovalently closed prior to intravenous injection by hybridization of the aptamers to their partially complementary strands. The binding of nucleolin to the aptamer triggered the conformation transition from duplex to intramolecular G-quadraplex and thereby induced mechanical opening of the origami nanotube and exposed the encapsulated thrombins, leading to the activation of coagulation at the tumor site and the inhibition of tumor growth.

\section{Spatial regulation of biomolecules and nanoparticles}

In addition to regulating the structural morphology of DNA nanostructures, the spatial regulation of any biomolecules and inorganic nanoparticles that can be anchored to DNA nanostructures can be achieved. This approach enormously extends the manipulation capacity, from regulating motions of materials fabricated solely from DNA to manipulating the distance-dependent properties of any object that can be connected to DNA. For instance, two gold nanorods were assembled upon two origami units into an L-shaped configuration that displayed strong chiroplasmonic signals (Fig. $4 \mathrm{a})^{38}$. When a G-quadruplex acts as the linker between the origami blocks, the interorigami/inter-rod distance can be adjusted by UV irradiation due to the use of an azobenzene derivative. It adopted the trans form under visible light and stabilized the G-quadruplex but switched to the cis form under UV light, which disassociated the DNA quadruplex structure. As such, DNA tetraplex conformational switching was subsequently transduced in situ into either enhanced or dampened chiral plasmonic signals.

Another feature of controlling enzyme activity via spatial regulation was demonstrated by using a DNA tetrahedron (Fig. 4b $)^{63}$. Specifically, in the design of an opening/closing-adjustable DNA tetrahedron, an i-motif and its partially mismatched complementary sequence constituted one of the six duplex edges, which disassembled at $\mathrm{pH}$ 6.4, thus opening the tetrahedron, and rehybridized at $\mathrm{pH} 7.3$, leading to the reformation of the tetrahedron. RNase A was encapsulated inside the cavity, and its enzyme activity was found to be reversibly controlled via $\mathrm{pH}$-dependent opening and closing of the DNA tetrahedron because the outer DNA cage limited its exposure to substrates.

In addition to controlling enzyme activity, the reconfiguration of three-dimensional DNA objects can be utilized to develop smart, targeted drug delivery systems that can respond to stimuli present in living organisms. For 
a

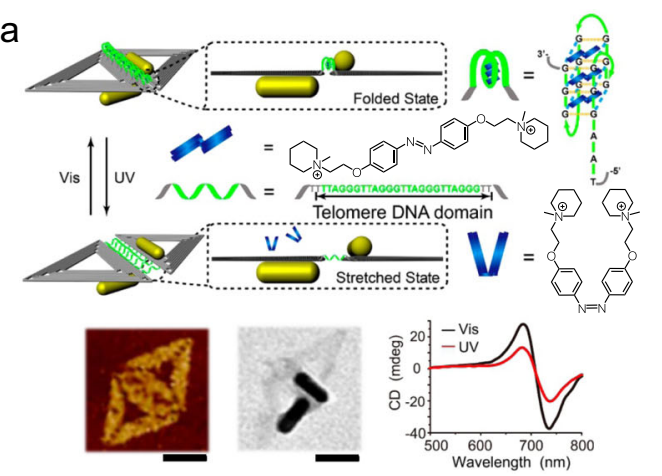

b

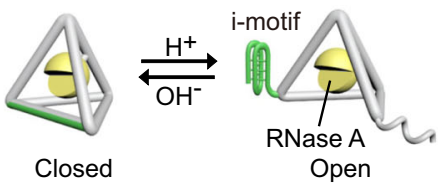

C

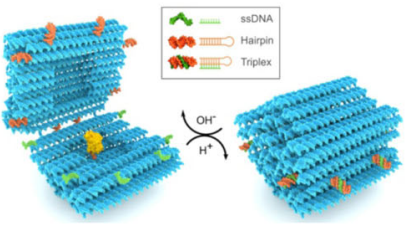

Fig. 4 Spatial manipulation of functional elements anchored on DNA nanostructures. a Photoregulation of gold nanorods plasmonic nanostructures based on the conformational switching of monomeric G-quadruplex induced by isomerization of an azobenzene derivative upon UV light irradiation. The inserted images at the bottom were an AFM image of the rhombus DNA origami, a TEM image of the L-shaped gold nanorods on the origami template, and photodependent CD spectra. Scale bars: $100 \mathrm{~nm}$. Reproduced with permission ${ }^{38}$. Copyright 2017 , American Chemical Society. b Enzyme activity regulation based on the reversible open and closure of DNA tetrahedron Reproduced with permission ${ }^{63}$. Copyright 2017 , American Chemical Society. c The reversible open and closure of a DNA origami nanocapsule containing a HRP payload controlled by eight pHresponsive latches which detached by existing in a hairpin and a ssDNA state at high pH, whereas connected by forming a triplex DNA at low pH. Reproduced with permission ${ }^{65}$. Copyright 2019, American Chemical Society.

instance, a DNA origami nanocapsule, with two capsule halves at one edge bearing sticky ends that are capable of associating into a bimolecular triplex, was demonstrated to undergo reversible opening/closing controlled by a sharp $\mathrm{pH}$ change, achieving the encapsulation and display of macromolecular payloads (Fig. 4c) ${ }^{64,65}$.

\section{Dynamic regulation of hierarchical DNA nanostructures}

Recently, research on the programmed assembly of hierarchical DNA architectures has attracted increasing attention $^{66}$. The emergence of different strategies to combine preformed structures into higher-order structures, such as sticky end association and blunt end stacking, broke through the limitation caused by the one-pot self-assembly method in scaling up both the size and complexity of DNA nanostructures ${ }^{67}$. However, these higher-order structures are inert, and the relative positions of their components are fixed once they are fabricated, which prevents their use in practical applications where reversible assembly/disassembly is critical $^{13}$. In this case, noncanonical nucleic acids provide a means to induce not only the assembly/ disassembly of DNA nanostructures but also rearrangement of the relative positions of the structural units ${ }^{33}$. This could be achieved in the sequence design stage by integrating the two strands of an intermolecular triplex/tetraplex conformation in the sticky ends protruding from the DNA nanostructures or by utilizing noncanonical DNA of intramolecular conformation and its partly complementary strand as the sticky ends. This form of dynamic regulation of hierarchical DNA nanostructures facilitates the development of intelligent DNA architectures, drug delivery systems, and molecular machines mimicking living systems to conduct conformational transitions in biomacromolecules to achieve specific functions according to the altered environment.

\section{Reversible assembly of hierarchical DNA nanostructures}

When multiple DNA nanostructures with tether strands comprising each of the two fragments of a bimolecular triplex/tetraplex are functionalized, they are able to transition reversibly between the aggregated and disaggregated states along with the hybridization and dissociation of the sticky ends ${ }^{32,33}$. Alternatively, by integration of a monomeric triplex/tetraplex-forming oligonucleotide in the sticky ends of one kind of DNA nanostructure and its partly complementary oligonucleotide in the sticky ends of another DNA nanostructure, the hybridization of the two types of sticky ends will bring together the two nanostructures, while the refolding of the triplex/tetraplex will lead to their separation ${ }^{32,33}$.

DNA origami was the most favorite substrate to employ to demonstrate the ability of a dimeric triplex/i-motif/Gquadruplex to assemble or disassemble multicomponent nanostructures by connecting or disconnecting individual structural units. For example, dimeric and monomeric origami structure transitions were conducted using an origami frame with a tether of ssDNA and another modified with a duplex (Fig. 5a) ${ }^{32}$. An 8-bp duplex (green) was incorporated in the design to stabilize the triplex structure.

In regard to applying a bimolecular DNA tetraplex to direct the assembly of DNA nanostructures, two means of adding directionality to their hybridization have been proven to be successful: appending a short duplex to the tetraplex or replacing the two loops of the tetraplex with well-designed hairpins. For instance, as shown in Fig. 5b, 


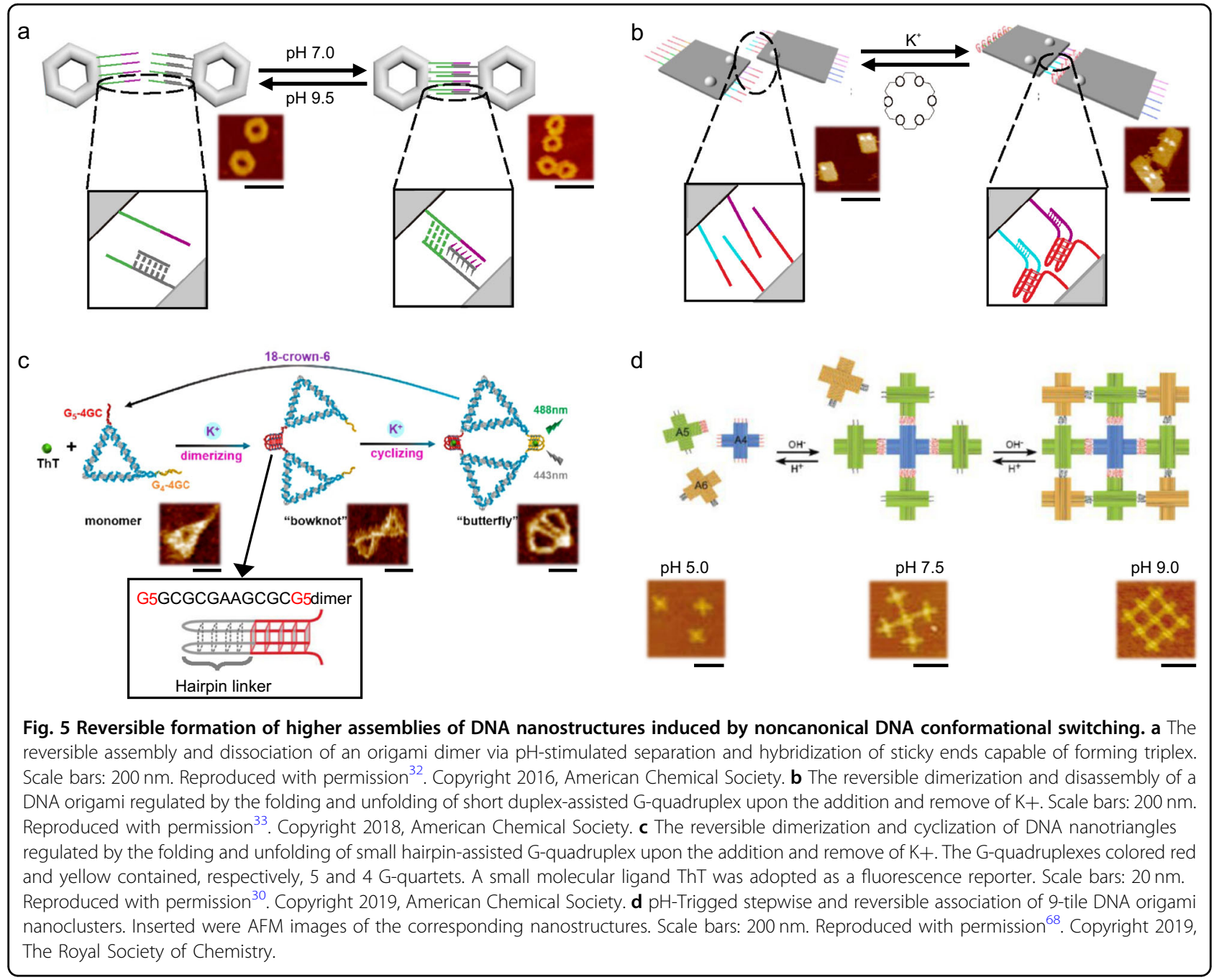

the alternating addition of $\mathrm{K}^{+}$and cation chelating agent 18-crown-6-ether will control dissociation and reassembly of the origami dimer ${ }^{33}$. The formation of the bimolecular G-quadruplex relied on the assistance of the short duplex (purple and cyan in the figure); otherwise, any two of the G-rich strands would hybridize and afford a mixture of disordered origami dimers.

Another example employed bimolecular G-quadruplexes modified with hairpins as two sets of sticky ends to perform the dimerization and cyclization of a triangular DNA nanostructure (Fig. 5c) ${ }^{30}$. In this instance, two G-rich strands with different stabilities, G $_{4}$ GCGCGAAGCGCG $_{4}$ (abbreviated as $\mathrm{G}_{4}-4 \mathrm{GC}$, yellow) and $\mathrm{G}_{5}$ GCGCGAAGC $\mathrm{GCG}_{5}$ (abbreviated as $\mathrm{G}_{5}-4 \mathrm{GC}$, red), were integrated on two vertexes of the DNA nanotriangle. With higher thermal stability and faster folding kinetics, G5-4GC folded in advance of G4-4GC, leading to the first formation of a bowknot-like structure and then cyclization. Unmodified G-rich sequences were not robust enough to induce the dimerization of the DNA triangles under the given conditions. Subjecting the cyclic product to a $\mathrm{K}^{+}$chelator 18-crown- 6 led to the reformation of the nanotriangle monomer.

In addition to dimerization, stepwise multimerization of DNA nanostructures could be achieved by incorporating multiple sets of sticky ends containing noncanonical nucleic acids. The sequential utilization of two triplexduplex conformational switching elements differing in base compositions was implemented to reversibly control the multimerization of DNA origami, mimicking living systems to assemble functional macromolecules in response to certain stimuli in a stepwise and reversible fashion $^{32,68}$. The $\mathrm{pH}$ sensitivity of the triplex depended strongly on the relative content of CGC/TAT triplets in the sequence. For instance, by increasing the TAT content from 0 to $100 \%$, the observed $\mathrm{pK}_{\mathrm{a}}$ for a 10 -bp-stem triplex was increased from 6.5 to $10.2^{34}$. Thus, the regulation of triplex $\mathrm{pH}$ sensitivity over a wide window of $\mathrm{pK}_{\mathrm{a}}$ variation $(\sim 5 \mathrm{pH}$ units) by manipulating the relative content of CGC/TAT triplets provided a means to realize the 

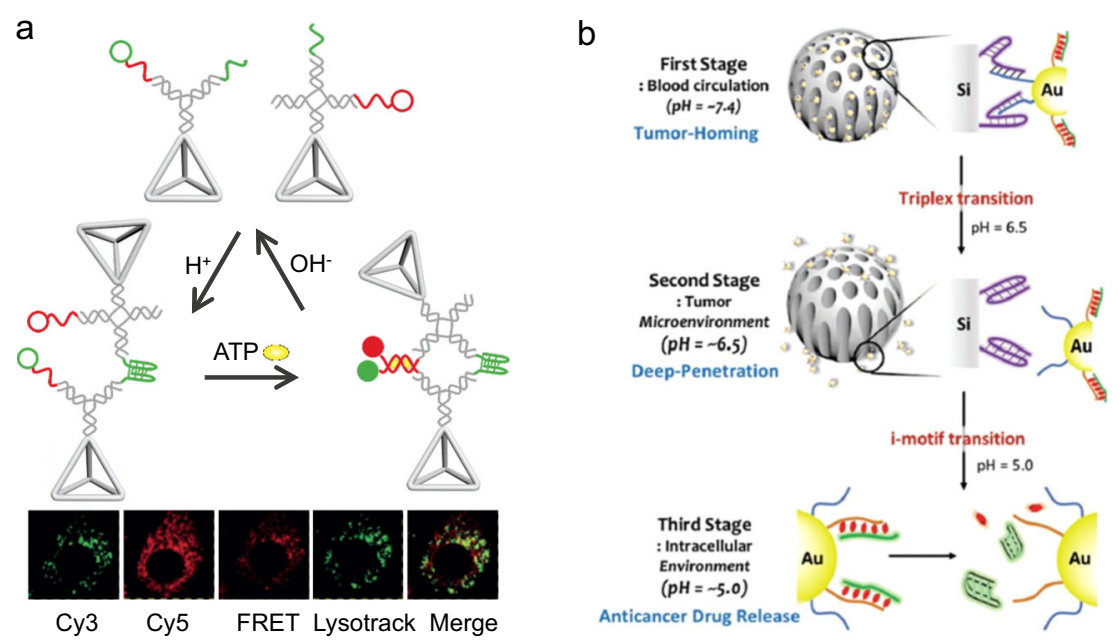

Fig. 6 Dynamic regulation of hierarchical DNA nanostructures for imaging and drug delivery. a Subcellular ATP imaging based on the dimerization of dimeric i-motif-functionalized DNA tetrahedron. The green and red circles denote respectively Cy3 and Cy5, and the gold ellipse denotes ATP. Below showed MCF-7 cells treated with the sensor for $4 \mathrm{~h}$. Colocalization of the tetrahedron dimer (red) and Lysotracker (green) was indicated by the yellow fluorescence signal after merging. Reproduced with permission ${ }^{31}$. Copyright 2019, Wiley-VCH. b Sequential disaggregation of a drug-loaded SNAcontaining hybrid nanoparticle in the penetrating route in tumor. Reproduced with permission ${ }^{70}$. Copyright 2018, Wiley-VCH.

hierarchical fabrication of complicated and/or large DNA structures reversibly and selectively in response to multiple steps of environmental stimulation, which would not be possible by duplex formation alone. In an example, origami with triplexes of varying $\mathrm{pK}_{\mathrm{a}}$ as linkers was subjected to transition among the monomer, dimer, and trimer states in a stepwise fashion in response to three different $\mathrm{pH}$ environments ${ }^{68}$. This strategy was further adopted in the stepwise construction of nine-unit DNA origami clusters (Fig. 5d).

In addition to DNA origami, other forms of nanostructures (DNA tetrahedron, SNA, etc.) can also be readily constructed into reversible hierarchical assemblies with noncanonical nucleic acids. Since the DNA tetrahedron was predominantly confined in the acidic organelle lysosome after endocytosis ${ }^{69}$, it is reasonable to implement the DNA tetrahedron in applications related to the acidic medium in lysosomes ( $\mathrm{pH} 4.5-5.0$ ), such as imaging biomolecules present exclusively in lysosomes. A recent study achieved lysosome-specific ATP imaging by implementing an AND-logic gate, with $\mathrm{H}^{+}$and ATP as inputs, on DNA tetrahedrons ${ }^{31}$. As depicted in Fig. 6a, one-half of a dimeric i-motif and one-half of a dimeric ATP aptamer were tethered to the vertexes of two tetrahedrons so that once the tetrahedrons entered acidic lysosomes, they assembled into a heterodimeric architecture by forming an intermolecular i-motif, thereby enabling proximity-induced ATP aptamer folding and FRET activation in response to endogenous ATP.

Moreover, the weak acidity (pH 6.4-6.9) of the tumor microenvironment becomes a valuable target in the design of controlled drug delivery systems. SNA assemblies loaded with antitumor drugs are able to release them by disaggregation in response to the acidic milieu. For example, a deep-penetrating drug delivery system was developed by employing stepwise triplex-duplex and imotif-duplex conformational switching, to direct the disaggregation of SNAs (Fig. 6b). ${ }^{70}$ Specifically, gold nanoparticles with a diameter of $\sim 15 \mathrm{~nm}$ were loaded into the pores of mesoporous silica nanoparticles (diameter $\approx$ $150 \mathrm{~nm}$ ) via triplex-forming sticky ends, and C-rich sticky ends in their duplex conformation embedded the anticancer drug doxorubicin into the surface of SNAs. When this type of drug carrier was administered, it first accumulated in the tumor region via enhanced permeability and retention effects. Subsequently, the slightly acidic tumor microenvironment $(\mathrm{pH} \approx 6.5)$ induced the structural transition of triplex DNA, leading to the release of SNAs from the silica nanoparticle pores to the deep region of the tumor tissue, because of their small size. After the SNAs were internalized into the tumor cells, the acidic endosomal $\mathrm{pH}$ (ca. $\mathrm{pH}$ 5.0) promoted the folding of the i-motif, which led to the final release of doxorubicin. This dual $\mathrm{pH}$ responsiveness based on the cooperative conformational switching of two types of noncanonical nucleic acids prompted the successful two-step disassembly of novel SNA-containing nanoparticles in vivo.

\section{Spatial rearrangement of hierarchical DNA nanostructures}

Taking advantage of the stimuli responsiveness of noncanonical nucleic acids, not only the controlled and reversible assembly of DNA nanostructures, but also their spatial rearrangement could be achieved. The two conformational transition strategies, namely, intermolecular- 


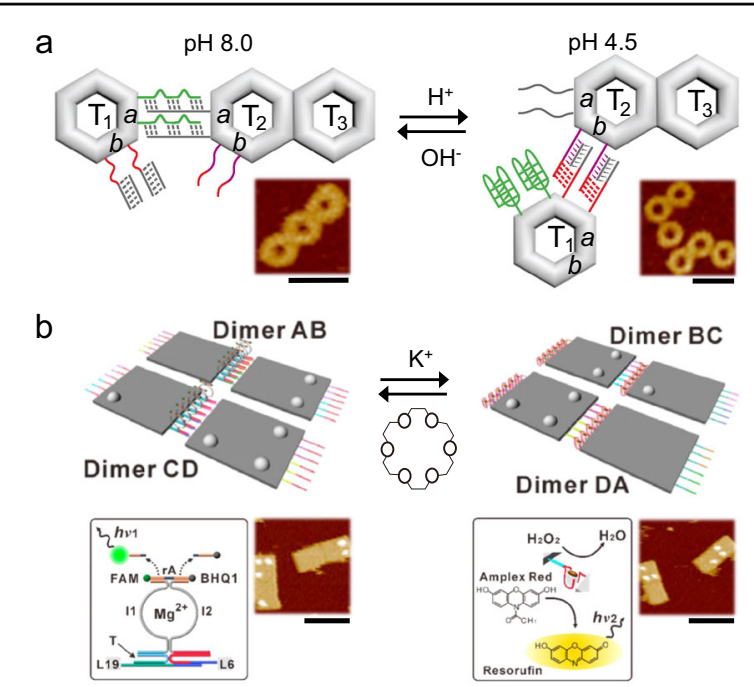

Fig. 7 Spatial rearrangement of DNA origami assemblies induced by noncanonical nucleic acid conformation switching. a Structural isomerization of a three frame DNA origami induced by $\mathrm{pH}$ variation. AFM images of the corresponding nanostructures were inserted. Scale bars correspond to $200 \mathrm{~nm}$. Reproduced with permission ${ }^{32}$. Copyright 2016, American Chemical Society. b A double replacement reaction demonstrated by DNA origami in which origami dimer mixture transitioned between $A B+C D$ and $B C+D A$ using $K+$ ions as triggers. Scale bars: $200 \mathrm{~nm}$. Reproduced with permission ${ }^{33}$. Copyright 2018, American Chemical Society.

and intramolecular-duplex conformational switches (as illustrated in Fig. 2), exhibited inverse effects in directing the aggregation and disassembly of DNA nanostructures, i.e., the introduction of a certain stimulus that connects structural units via an intermolecular conformational transition strategy, and dissociate them via an intramolecular-duplex transition strategy. It is therefore advantageous to employ both strategies in a system requiring alternating base-pairing and disassociation of two sets of sticky ends. For instance, combinatory utilization of these strategies realized 'isomerization' of origami assemblies, by considering each origami unit as an 'atom' and their assemblies as 'molecules ${ }^{32}$. Specifically, as depicted in Fig. $7 \mathrm{a}$, in the $\mathrm{T}_{1}-a$ edge- $\mathrm{T}_{2}-a$ edge 'bond', an i-motif-forming sequence (green) and its partly complementary sequence functioned as sticky ends, while in the $\mathrm{T}_{1}-b$ edge- $\mathrm{T}_{2}-b$ edge 'bond', two halves of a split triplex were included in the sticky ends. Alkaline conditions allowed the existence of only a duplex (hence the bonding of the $\mathrm{T}_{1}-a$ edge- $\mathrm{T}_{2}-a$ edge) and prohibited $\mathrm{i}$-motif and triplex formation, while acidic conditions stabilized both the intra-i-motif and intertriplex, leading to the cleavage of the $\mathrm{T}_{1}-a$ edge- $\mathrm{T}_{2}-a$ edge 'bond' and the formation of the $\mathrm{T}_{1}-b$ edge- $\mathrm{T}_{2}-b$ edge 'bond'. In this way, the trimerorigami structure was able to transition between linear and angular 'isomers' upon $\mathrm{pH}$ stimulation.
More complicated reconfiguration of origami assemblies was realized by using four sets of sticky ends, two of which were able to switch between an intermolecular tetraplex and a random coil (e.g., dimer DA and dimer BC in Fig. 7b), while the other two, between an intramolecular tetraplex and a duplex (e.g., dimer $\mathrm{AB}$ and dimer $\mathrm{CD})^{33}$. The alternating addition of $\mathrm{K}^{+}$and cation chelating agent 18-crown-6-ether controlled the dissociation and reassembly of the origami dimer. This type of stimulation-mediated morphological interconversion laid the foundation of the 'origami chemistry' concept introduced by Willner's group, which considers individual origami tiles as reaction elements to be subjected to synthesis, decomposition, single replacement, and double replacement. Well-designed noncanonical nucleic acid sequences were integrated in the protruding tethers on the tiles to connect and disconnect reaction elements on demand. The reconfiguration of the $\mathrm{AB}+\mathrm{CD}$ dimer mixture into $\mathrm{BC}+\mathrm{DA}$ shown in Fig. $7 \mathrm{~b}$ resembles a double-replacement reaction. Moreover, a switchable catalytic function was engineered by introducing sequences of $\mathrm{Mg}^{2+}$-ion-dependent DNAzyme and hemin/ G-quadruplex DNAzyme in the bridge.

\section{Conclusions}

The past few years have seen the intriguing interweaving of noncanonical nucleic acids with artificial DNA architectures. In this review, we highlighted different strategies for subjecting noncanonical nucleic acids to conformational transitions and their utilization in regulating DNA nanostructures for nanomedical applications. The specific roles of both entities were examined in every surveyed example. The incorporation of noncanonical nucleic acids into predesigned DNA nanostructures renders successful manipulation of the morphological alteration, movement, and assembly/disassembly processes of static nanostructures, exhibiting potential in fabricating biomimetic materials, molecular motors, and controlled drug delivery systems.

With the accumulation of experience in integrating noncanonical nucleic acids into DNA architectures, some previously neglected issues have come to light. For example, the stability of noncanonical nucleic acids varies to a noticeable extent after they are incorporated into DNA nanostructures ${ }^{71,72}$. Careful examination of their thermodynamics and kinetics is thus recommended when they become components of a larger structure. Additionally, the exact folding topology of DNA tetraplexes has rarely been taken into consideration in most designs, probably on account of their complexity. Nonetheless, differences in topology may influence the morphology, thermodynamics, and kinetics of the synthesized DNA nanostructure ${ }^{27}$. A deeper understanding of the correlation between the DNA 
primary structure and its folding topology will prompt the utilization of DNA tetraplexes with a single topology in delicate devices ${ }^{27,42}$.

Although various studies have been carried out to examine the ability of noncanonical nucleic acids to transform artificial DNA nanostructures, there remains a need for a new approach to regulate the motion and assembly process of the existing systems. It is highly expected that more manipulation capacities will come from introducing small molecule ligands or proteins that specifically interact with noncanonical nucleic acids, or from introducing appropriately chemically modified nucleosides $^{73}$, as well as manmade DNA analogs (locked nucleic acids, unlocked nucleic acids, peptide nucleic acids, etc. $)^{74,75}$.

The regulatory capacity of noncanonical DNA on DNA nanostructures holds promising prospects in the fields of early cancer diagnosis and synthetic biology ${ }^{76}$. For example, a two-state cage-like DNA nanostructure, which can be opened and closed by noncanonical DNA, could serve as a platform for the capture and release of diagnostic biomarkers (e.g., microRNA, circulating tumor DNA, proteins, and extracellular vesicles) and for circulating tumor cells ${ }^{77}$ in a repeatable and on-demand manner. When the DNA nanostructure is immobilized on the interface of either a solid or soft material (e.g., gold surface, microfluidic chip, or hydrogel), these biomarkers could be extracted from body fluids and then enriched for further analysis. On the other hand, living cells can be conjugated with artificial DNA architectures ${ }^{78}$, and it is therefore possible to expand the spatial arrangement or assembly/disassembly information of the DNA architectures to cells. This would be advantageous to the engineering of cellular morphology and intercellular communication $^{79}$.

Overall, the richness of nucleic acid conformational polymorphisms, the versatility of artificial DNA nanostructures, and the interweaving of the two groups synchronously contribute to the growth of DNA-related science and technology, generating an ever-increasing ability to perform manipulation at the nanoscale. The resultant opportunities in developing biomimicking materials, molecular motors, drug delivery systems, and biosensors hold great promise for translating DNA materials and devices from the laboratory to the clinic in the near future.

\section{Acknowledgements}

This work was financially supported by the National Natural Science Foundation of China $(21804088,21902101,21834007,21991134)$, the National Key R\&D Program of China (2018YFA0902601), the Shanghai Pujiang Program (19PJ1404700), the Natural Science Foundation of Shanghai (20ZR1425600) and the Shanghai Key Laboratory for Nucleic Acid Chemistry and Nanomedicine (2020ZYB004).
Conflict of interest

The authors declare no competing interests.

\section{Publisher's note}

Springer Nature remains neutral with regard to jurisdictional claims in published maps and institutional affiliations.

Received: 16 November 2020 Revised: 5 March 2021 Accepted: 29 March 2021.

Published online: 30 April 2021

\section{References}

1. Seeman, N. C. Nucleic acid junctions and lattices. J. Theor. Biol. 99, 237-247 (1982).

2. Chen, J. \& Seeman, N. C. Synthesis from DNA of a molecule with the connectivity of a cube. Nature 350, 631-633 (1991).

3. Goodman, R. P. et al. Rapid chiral assembly of rigid DNA building blocks for molecular nanofabrication. Science 310, 1661-1665 (2005).

4. linuma, R. et al. Polyhedra self-assembled from DNA tripods and characterized with 3D DNA-PAINT. Science 344, 65-69 (2014).

5. Rothemund, P. W. K. Folding DNA to create nanoscale shapes and patterns. Nature 440, 1-82 (2006)

6. Xiong, R., Grant, A. M., Ma, R., Zhang, S. \& Tsukruk, V. V. Naturally-derived biopolymer nanocomposites: interfacial design, properties and emerging applications. Mater. Sci. Eng. R. Rep. 125, 1-41 (2018).

7. Cutler, J. I., Auyeung, E. \& Mirkin, C. A. Spherical nucleic acids. J. Am. Chem. Soc. 134, 1376-1391 (2012).

8. Ge, Z., Gu, H., Li, Q. \& Fan, C. Concept and development of framework nucleic acids. J. Am. Chem. Soc. 140, 17808-17819 (2018).

9. Hong, F., Zhang, F. Liu, Y. \& Yan, H. DNA origami: scaffolds for creating higher order structures. Chem. Rev. 117, 12584-12640 (2017).

10. Ramezani, H. \& Dietz, H. Building machines with DNA molecules. Nat. Rev. Genet. 21, 5-26 (2020).

11. Surana, S., Shenoy, A. R. \& Krishnan, Y. Designing DNA nanodevices for compatibility with the immune system of higher organisms. Nat. Nanotechnol. 10, 741-747 (2015).

12. Deluca, M., Shi, Z., Castro, C. E. \& Arya, G. Dynamic DNA nanotechnology: toward functional nanoscale devices. Nanoscale Horiz. 5, 182-201 (2020).

13. Liu, X., Lu, C. H. \& Willner, I. Switchable reconfiguration of nucleic acid nanostructures by stimuli-responsive DNA machines. Acc. Chem. Res. 47, 1673-1680 (2014).

14. Yao, G. et al. Programming nanoparticle valence bonds with single-stranded DNA encoders. Nat. Mater. 19, 781-788 (2020).

15. Rajagopal, P. \& Feigon, J. Triple-strand formation in the homopurine:homopyrimidine DNA oligonucleotides $\mathrm{d}(\mathrm{G}-\mathrm{A})_{4}$ and $\mathrm{d}(\mathrm{T}-\mathrm{C})_{4}$. Nature $\mathbf{3 3 9}$, 637-640 (1989).

16. Parkinson, G. N., Lee, M. P. H. \& Neidle, S. Crystal structure of parallel quadruplexes from human telomeric DNA. Nature 417, 876-880 (2002).

17. Gehring, K., Leroy, J. L. \& Guéron, M. A tetrameric DNA structure with protonated cytosine-cytosine base pairs. Nature 363, 561-565 (1993).

18. Neidle, S. Principles of Nucleic Acid Structure (Elsevier Inc., 2008).

19. Buske, F. A., Mattick, J. S. \& Bailey, T. L. Potential in vivo roles of nucleic acid triple-helices. RNA Biol. 8, 427-439 (2011).

20. Biffi, G., Tannahill, D., McCafferty, J. \& Balasubramanian, S. Quantitative visualization of DNA G-quadruplex structures in human cells. Nat. Chem. 5, 182-186 (2013).

21. Zeraati, M. et al. I-motif DNA structures are formed in the nuclei of human cells. Nat. Chem. 10, 631-637 (2018).

22. Tateishi-Karimata, H. \& Sugimoto, N. Chemical biology of non-canonical structures of nucleic acids for therapeutic applications. Chem. Commun. 56, 2379-2390 (2020)

23. Rhee, S., Han, Z. J., Liu, K., Miles, H. T. \& Davies, D. R. Structure of a triple helical DNA with a triplex-duplex junction. Biochemistry 38, 16810-16815 (1999).

24. Wärmländer, S., Sandström, K., Leijon, M. \& Gräslund, A. Base-pair dynamics in an antiparallel DNA triplex measured by catalyzed imino proton exchange monitored via ' $\mathrm{H}$ NMR Spectroscopy. Biochemistry $\mathbf{4 2}$, 12589-12595 (2003) 
25. James, P. L., Brown, T. \& Fox, K. R. Thermodynamic and kinetic stability of intermolecular triple helices containing different proportions of $\mathrm{C}^{+} \cdot \mathrm{GC}$ and T.AT triplets. Nucleic Acids Res. 31, 5598-5606 (2003).

26. Wu, P., Kawamoto, Y., Hara, H. \& Sugimoto, N. Effect of divalent cations and cytosine protonation on thermodynamic properties of intermolecular DNA double and triple helices. J. Inorg. Biochem. 91, 277-285 (2002).

27. Li, T. \& Famulok, M. I-motif-programmed functionalization of DNA nanocircles. J. Am. Chem. Soc. 135, 1593-1599 (2013).

28. Zhou, J., Bourdoncle, A., Rosu, F., Gabelica, V. \& Mergny, J. L. Tri-G-quadruplex: controlled assembly of a G-quadruplex structure from three G-rich strands. Angew. Chem. Int. Ed. 51, 11002-11005 (2012).

29. Zhang, N., Phan, A. T. \& Patel, D. J. (3+1) Assembly of three human telomeric repeats into an asymmetric dimeric G-quadruplex. J. Am. Chem. Soc. 127, 17277-17285 (2005).

30. Zheng, J. et al. Ultrastable bimolecular G-quadruplexes programmed DNA nanoassemblies for reconfigurable biomimetic DNAzymes. ACS Nano 13, 11947-11954 (2019).

31. Peng, P., Du, Y., Zheng, J., Wang, H. \& Li, T. Reconfigurable bioinspired framework nucleic acid nanoplatform dynamically manipulated in living cells for subcellular imaging. Angew. Chem. Int. Ed. 58, 1648-1653 (2019).

32. Wu, N. \& Willner, I. pH-Stimulated reconfiguration and structural isomerization of origami dimer and trimer systems. Nano Lett. 16, 6650-6655 (2016).

33. Wang, J., Yue, L., Wang, S. \& Willner, I. Triggered reversible reconfiguration of Gquadruplex-bridged 'domino'-type origami dimers: application of the systems for programmed catalysis. ACS Nano 12, 12324-12336 (2018).

34. Idili, A., Vallée-Bélisle, A. \& Ricci, F. Programmable pH-triggered DNA nanoswitches. J. Am. Chem. Soc. 136, 5836-5839 (2014).

35. Lan, P., Tran, T., Mergny, J. \& Alberti, P. Stability of telomeric G-quadruplexes. Nucleic Acids Res. 39, 3282-3294 (2011).

36. Nonin, S., Phan, A. T. \& Leroy, J. L. Solution structure and base pair opening kinetics of the i-motif dimer of d(5mCCTTACC): a noncanonical structure with possible roles in chromosome stability. Structure 5, 1231-1247 (1997).

37. Shi, L., Peng, P., Du, Y. \& Li, T. Programmable i-motif DNA folding topology for a pH-switched reversible molecular sensing device. Nucleic Acids Res. 45, 4306-4314 (2017).

38. Jiang, Q. et al. Stimulus-responsive plasmonic chiral signals of gold nanorods organized on DNA origami. Nano Lett. 17, 7125-7130 (2017)

39. Yang, S., Liu, W., Nixon, R. \& Wang, R. Metal-ion responsive reversible assembly of DNA origami dimers: G-quadruplex induced intermolecular interaction. Nanoscale 10, 3626-3630 (2018).

40. Suzuki, Y., Kawamata, I., Mizuno, K. \& Murata, S. Large deformation of a DNAorigami nanoarm induced by the cumulative actuation of tension-adjustable modules. Angew. Chem. Int. Ed. 59, 6230-6234 (2020).

41. Shikiya, R. \& Marky, L. A. Calorimetric unfolding of intramolecular triplexes: length dependence and incorporation of single AT - \$ TA substitutions in the duplex domain. J. Phys. Chem. B 109, 18177-18183 (2005).

42. Karsisiotis, A. I., O'Kane, C. \& Webba da Silva, M. DNA quadruplex folding formalism - a tutorial on quadruplex topologies. Methods 64, 28-35 (2013).

43. Chandrasekaran, A. R. \& Rusling, D. A. Triplex-forming oligonucleotides: a third strand for DNA nanotechnology. Nucleic Acids Res. 46, 1021-1037 (2018).

44. Zhou, J. et al. Formation of i-motif structure at neutral and slightly alkaline $\mathrm{pH}$. Mol. Biosyst. 6, 580-586 (2010).

45. Nguyen, T., Fraire, C. \& Sheardy, R. D. Linking $\mathrm{pH}$, temperature, and $\mathrm{K}^{+}$concentration for DNA i-motif formation. J. Phys. Chem. B 121, $7872-7877$ (2017).

46. Mullen, M. A., Assmann, S. M. \& Bevilacqua, P. C. Toward a digital gene response: RNA G-quadruplexes with fewer quartets fold with higher cooperativity. J. Am. Chem. Soc. 134, 812-815 (2012).

47. Day, H. A., Huguin, C. \& Waller, Z. A. E. Silver cations fold i-motif at neutral pH. Chem. Commun. 49, 7696-7698 (2013).

48. Mergny, J. L., Lacroix, L., Hélène, C., Han, X. \& Leroy, J. L. Intramolecular folding of pyrimidine oligodeoxynucleotides into an i-DNA motif. J. Am. Chem. Soc. 117, 8887-8898 (1995).

49. Floris, R., Scaggiante, B., Manzini, G., Quadrifoglio, F. \& Xodo, L. E. Effect of cations on purine-purine-pyrimidine triple helix formation in mixed-valence salt solutions. Eur. J. Biochem. 260, 801-809 (1999).

50. Dong, Y., Yang, Z. \& Liu, D. DNA nanotechnology based on i-motif structures. Acc. Chem. Res. 47, 1853-1860 (2014).

51. Majikes, J. M., Ferraz, L. C. C. \& LaBean, T. H. pH-Driven actuation of DNA origami via parallel i-motif sequences in solution and on surfaces. Bioconjug. Chem. 28, 1821-1825 (2017).
52. Zhu, D. et al. A surface-confined proton-driven DNA pump using a dynamic 3D DNA scaffold. Adv. Mater. 28, 6860-6865 (2016).

53. Webb, B. A., Chimenti, M., Jacobson, M. P. \& Barber, D. L. Dysregulated pH: a perfect storm for cancer progression. Nat. Rev. Cancer 11, 671-677 (2011).

54. Leung, K. H., Chakraborty, K., Saminathan, A. \& Krishnan, Y. A DNA nanomachine chemically resolves lysosomes in live cells. Nat. Nanotechnol. 14, 176-183 (2019).

55. Krishnan, Y., Zou, J. \& Jani, M. S. Quantitative imaging of biochemistry in situ and at the nanoscale. ACS Cent. Sci. 6, 1938-1954 (2020).

56. Yang, L. et al. Direct fluorescent detection of blood potassium by ion-selective formation of intermolecular G-quadruplex and ligand binding. Anal. Chem. 88, 9285-9292 (2016)

57. Ma, D., Wang, W., Mao, Z., Kang, T. \& Han, Q. Utilization of G-quadruplexforming aptamers for the construction of luminescence sensing platforms. ChemPlusChem 82, 8-17 (2017).

58. Zhou, Y. J. et al. Molecular switching of a self-assembled 3D DNA nanomachine for spatiotemporal $\mathrm{pH}$ mapping in living cells. Anal. Chem. 91, 10366-10370 (2019)

59. Ke, Y., Meyer, T., Shih, W. M. \& Bellot, G. Regulation at a distance of biomolecular interactions using a DNA origami nanoactuator. Nat. Commun. 7, 1-8 (2016).

60. Li, J., Jiao, Y., Liu, Q. \& Chen, Z. Colorimetric detection of thrombin based on intensity of gold nanoparticle oligomers with dark-field microscope. ACS Sustain. Chem. Eng. 6, 6738-6745 (2018).

61. Chai, H. et al. Fabrication of polymeric ferrocene nanoparticles for electrochemical aptasensing of protein with target-catalyzed hairpin assembly. Anal. Chem. 91, 9940-9945 (2019).

62. Li, S. et al. A DNA nanorobot functions as a cancer therapeutic in response to a molecular trigger in vivo. Nat. Biotechnol. 36, 258-264 (2018).

63. Kim, S. H. et al. Reversible regulation of enzyme activity by $\mathrm{pH}$-responsive encapsulation in DNA nanocages. ACS Nano 11, 9352-9359 (2017).

64. ljäs, H. et al. origami nanocapsule for $\mathrm{pH}$-controlled encapsulation and display of cargo. ACS Nano 13, 5959-5967 (2019).

65. Tan, X., Jia, F., Wang, P. \& Zhang, K. Nucleic acid-based drug delivery strategies. J. Control. Release 323, 240-252 (2020).

66. Ong, L. L. et al. Programmable self-assembly of three-dimensional nanostructures from 10,000 unique components. Nature 552, 72-77 (2017).

67. Lin, T. et al. Hierarchical assembly of DNA nanostructures based on four-way toehold-mediated strand displacement. Nano Lett. 18, 4791-4795 (2018).

68. Yang, S., Liu, W. \& Wang, R. Control of the stepwise assembly-disassembly of DNA origami nanoclusters by $\mathrm{pH}$ stimuli-responsive DNA triplexes. Nanoscale 11, 18026-18030 (2019).

69. Liang, L. et al. Single-particle tracking and modulation of cell entry pathways of a tetrahedral DNA nanostructure in live cells. Angew. Chem. Int. Ed. 53, 7745-7750 (2014).

70. Kim, J. et al. Programmed nanoparticle-loaded nanoparticles for deeppenetrating 3D cancer therapy. Adv. Mater. 30, 1-8 (2018).

71. Mao, X. et al. Activity modulation and allosteric control of a scaffolded DNAzyme using a dynamic DNA nanostructure. Chem. Sci. 7, 1200-1204 (2016).

72. Ottaviani, A. et al. Engineering a responsive DNA triple helix into an octahedral DNA nanostructure for a reversible opening/closing switching mechanism: a computational and experimental integrated study. Nucleic Acids Res. 46, 9951-9959 (2018)

73. Halder, S. \& Krishnan, Y. Design of ultrasensitive DNA-based fluorescent pH sensitive nanodevices. Nanoscale 7, 10008-10012 (2015).

74. Berger, O. \& Gazit, E. Molecular self-assembly using peptide nucleic acids. Biopolymers 108, 1-6 (2017).

75. Modi, S., Wani, A. H. \& Krishnan, Y. The PNA-DNA hybrid i-motif: implications for sugar-sugar contacts in i-motif tetramerization. Nucleic Acids Res. 34, 4354-4363 (2006).

76. Broza, Y. Y. et al. Disease detection with molecular biomarkers: from chemistry of body fluids to nature-inspired chemical sensors. Chem. Rev. 119, 11761-11817 (2019)

77. Jaworski, J. J., Morgan, R. D. \& Sivakumar, S. Circulating cell-free tumour DNA for early detection of pancreatic cancer. Cancers 12, 1-16 (2020).

78. Ge, Z. et al. Programming cell-cell communications with engineered cell origami clusters. J. Am. Chem. Soc. 142, 8800-8808 (2020).

79. Gartner, Z. J., Prescher, J. A. \& Lavis, L. D. Unraveling cell-to-cell signaling networks with chemical biology. Nat. Chem. Biol. 13, 564-568 (2017). 